\title{
Bactericidal/Permeability-Increasing Fold-Containing Family B Member 4 May Be Associated with NSAID-Induced Enteropathy
}

\author{
Shunji Fujimori ${ }^{1}$ (D) Koya Fukunaga ${ }^{2} \cdot$ Atsushi Takahashi $^{2,3} \cdot$ Taisei Mushiroda $^{2} \cdot$ Michiaki Kubo $^{2} \cdot$ Ryuzo Hanada $^{4}$. \\ Mari Hayashida ${ }^{5} \cdot$ Toshiyuki Sakurai $^{6} \cdot$ Katsuhiko Iwakiri $^{1} \cdot$ Choitsu Sakamoto $^{1}$
}

Received: 5 July 2018 / Accepted: 19 October 2018 / Published online: 30 October 2018

(c) The Author(s) 2018

\begin{abstract}
Background There is considerable individual variability in nonsteroidal anti-inflammatory drug (NSAID)-induced enteropathy.

Aim To identify the SNP that is most significantly involved with NSAID-induced enteropathy.

Methods One hundred fifty human subjects who were known to have a certain degree of loxoprofen- or celecoxib-induced small-intestinal damage from a previous study were enrolled. The subjects were divided into groups based on treatments and also on the increased number of small intestinal mucosal breaks. The candidate SNP was selected by an initial analysis of GWAS among the groups in various combinations. After the initial analysis, the gene including the specified SNP was analyzed in detail using GWAS and genotype imputation.

Results After analysis, 70 subjects receiving the loxoprofen treatment and 69 subjects receiving celecoxib treatment were determined to be eligible for the analysis. The minimum $p$ value in GWAS was detected in the analysis of 16 cases with an increase of five or more mucosal breaks and 123 controls with zero to four mucosal breaks. In the GWAS, five SNPs in the bactericidal/permeability-increasing fold-containing family B member 4 (BPIFB4) gene showed the lowest $p$ value $\left(p=2.69 \times 10^{-7}\right.$ with an odds ratio of 40.9$)$. Of the five SNPs, four were nonsynonymous SNPs (rs2070325: V268I, rs2889732: T320N, rs11699009: F527L, rs11696307: T533I, and rs11696310: intronic). Furthermore, 23 SNPs in BPIFB4 detected by genotype imputation based on the GWAS data also showed suggestive associations $\left(p<1 \times 10^{-6}\right)$.

Conclusion The results indicate that SNPs in BPIFB4 were associated with NSAID-induced small intestinal mucosal injury (UMIN: 000007936).
\end{abstract}

Keywords Small intestinal injury $\cdot$ Enteropathy $\cdot$ NSAID $\cdot$ GWAS $\cdot$ BPI $\cdot$ Capsule endoscopy

$\begin{array}{llll}\text { Abbreviations } & \text { OR } & \begin{array}{l}\text { Odds ratio } \\ \text { BPIFB4 }\end{array} \\ \begin{array}{ll}\text { Bactericidal/permeability-increasing fold-con- } \\ \text { taining family B member 4 }\end{array} & \begin{array}{l}\text { PPI } \\ \text { SNP }\end{array} & \begin{array}{l}\text { Proton pump inhibitor } \\ \text { Single nucleotide polymorphism }\end{array} \\ \text { GWAS } & \begin{array}{l}\text { Genome-wide association study } \\ \text { LD }\end{array} & \text { Linkage disequilibrium } & \\ \text { NSAID } & \text { Nonsteroidal anti-inflammatory drug } & & \end{array}$

Electronic supplementary material The online version of this article (https://doi.org/10.1007/s10620-018-5349-0) contains supplementary material, which is available to authorized users.

Shunji Fujimori

s-fujimori@nms.ac.jp

1 Department of Gastroenterology, Graduate School of Medicine, Nippon Medical School, 1-1-5, Sendagi, Bunkyo-ku, Tokyo 113-8603, Japan

2 RIKEN Center for Integrative Medical Sciences, Yokohama, Japan
3 Department of Genomic Medicine, Research Institute, National Cerebral and Cardiovascular Center, Osaka, Japan

4 Sumida Hospital, SOUSEIKAI, Tokyo, Japan

5 The Third Department of Internal Medicine, Kyorin University School of Medicine, Tokyo, Japan

6 Department of Gastroenterology and Hepatology, National Center for Global Health and Medicine, Tokyo, Japan 


\section{Introduction}

The capsule endoscopy-advanced modality was developed in 2000 and allows for full investigation of the entire small intestine. This technique revealed that nonsteroidal antiinflammatory drugs (NSAIDs) cause many small intestinal ulcerative lesions. Graham et al. [1] report that small bowel injury is observed in $71 \%$ of NSAID users compared with $10 \%$ of nonusers based on capsule endoscopy. At nearly the same time, Maiden et al. [2] report that capsule endoscopy shows new pathology in $68 \%$ of subjects after 14 days of treatment of the traditional NSAID diclofenac and with the proton pump inhibitor (PPI) omeprazole. According to these reports, the significance of small intestinal injury is increasingly important, particularly for the use of NSAIDs with the concomitant administration of PPIs for gastroprotection, which causes small intestinal injuries in 50-70\% of subjects [3-5].

The authors identified drugs to protect small intestinal mucosa from NSAID-induced injuries. First, we showed that coadministration of misoprostol, a prostaglandin analog, reduced the incidence of small intestinal mucosal breaks induced by a 2 -week administration of diclofenac with concomitant omeprazole administration in a singleblind randomized controlled trial (RCT) [6]. Next, we also showed that coadministration of the cytoprotective antiulcer agent rebamipide can reduce the intensity of small intestinal injury caused by the same diclofenac treatment as described previously in a double-blind RCT [7]. These studies demonstrate that NSAID treatment causes hundreds of small intestinal mucosal breaks in a few subjects. By contrast, the studies also indicate that treatment causes no small intestinal mucosal breaks in approximately $50 \%$ of subjects. In fact, there is considerable individual variability in NSAID-induced small intestinal injury [5]. There is a high possibility of the presence of a genetic factor that influences NSAID-induced small intestinal injury.

Drug-induced side effects are often associated with genetic polymorphisms. Several studies have evaluated the association of genetic polymorphisms with NSAIDinduced gastrointestinal injuries. The studies reported that single-nucleotide polymorphisms (SNPs) of the metabolic enzymes of NSAIDs, such as CYP2C9, CYP3A4, and prostaglandin synthesizing enzyme cyclooxygenase- 1 (COX-1) were significantly associated with gastrointestinal bleeding and/or ulceration [8, 9]. However, the studies investigated only a few candidate SNPs in the enzymes that metabolize NSAIDs and the arachidonic acid cascade. Therefore, a comprehensive analysis is necessary to identify other unknown SNPs showing stronger effects on NSAID-induced small intestinal injury than the previously reported SNPs.
A genome-wide association study (GWAS) is an exhaustive genetic polymorphism investigation to resolve the problem of studies analyzing only a few candidate SNPs. A GWAS makes it possible to identify loci associated with drug-induced side effects, which are unpredictable SNPs using a conventional candidate gene approach. Evaluating drug-induced side effects under identical conditions in terms of subjects, dosage amount, and duration of administration is preferable. In the clinical setting, it is difficult to achieve identical conditions because patients with a variety of diseases are administered various types of drugs over varying periods of time. Therefore, the authors performed a further GWAS analysis to clarify NSAID-induced small intestinal injury using subjects from a study we previously reported to compare small intestinal mucosal breaks between celecoxib monotherapy and concomitant treatment with loxoprofen and lansoprazole in healthy subjects. These patients were used because the study subjects were identical healthy subjects receiving the same dosage amount and had the same duration of administration [10]. The purpose of this study was to identify the SNP that was involved in susceptibility of NSAID-induced small intestinal mucosal injury.

\section{Subjects and Methods}

The study subjects were obtained from a previous study that the authors conducted to compare the number of small intestinal mucosal breaks between loxoprofen (a traditional NSAID) concomitant with lansoprazole (a PPI) and celecoxib (a COX-2 selective inhibitor) after a 14-day treatment. The details of the study were reported by Fujimori et al. [10]. Genomic analyses using DNA samples from the study subjects were performed. This study was approved by the ethics committee of Nippon Medical School, Kyushu Clinical Pharmacology Research Clinic Institutional Review Board, and RIKEN Center for Integrative Medical Sciences. This study was registered at the UMIN registry (ID: 000007936).

\section{Study Subjects}

One hundred fifty subjects were selected as eligible from the 230 healthy candidates screened by medical checks, including physical tests, laboratory tests, and interviews of medical history from April 2012 to March 2013. All subjects provided written informed consent for this study before undergoing baseline capsule endoscopy. The study subjects were required to be healthy Japanese volunteers aged 40-70 years old at the time of informed consent. "Healthy" was defined as (1) receiving no treatment and (2) having no clinically relevant abnormalities determined from the detailed medical history and full physical examination, 
including blood pressure, pulse rate measurement, 12-lead electrocardiogram, laboratory tests, and fecal occult blood test at screening. Subjects with a history of gastrointestinal ulcer, gastrointestinal operation, malignancy, cardiac disease, cerebrovascular disease, known hypersensitivity or allergy to celecoxib, aspirin, other NSAIDs or lansoprazole, gastrointestinal disease within 1 month or current gastrointestinal symptoms at the time of admission to the study, and subjects who were being treated with NSAIDs, including aspirin, anti-ulcer medications, antacids, steroids, or antibiotics within 1 month prior to the first day of the study were excluded.

\section{Protocol of the Previous Study}

All eligible subjects were randomly divided into two groups, a celecoxib group and a loxoprofen group. Age, gender, and Helicobacter pylori infection were equally distributed. After randomization, baseline capsule endoscopy was performed. After baseline capsule endoscopy, subjects in the celecoxib group were administered a celecoxib capsule $(100 \mathrm{mg}$ ) twice a day and lactose placebo capsule twice a day. Subjects in the loxoprofen group were given a loxoprofen capsule $(60 \mathrm{mg})$ three times a day and lansoprazole capsule $(15 \mathrm{mg})$ once a day. Small intestinal injuries were evaluated using PillCam ${ }^{\circledR}$ SB2 capsule endoscopy (Covidien, Dublin, Ireland). Baseline capsule endoscopies were performed at day 1. Post-treatment capsule endoscopies were performed within 3 days after completion of the drug regimen in all subjects who completed the study. All video image files were anonymized and evaluated by three skilled reviewers who did not have any information about the subjects. A mucosal break was defined as mucosal deficits in the small intestine with slough surrounded by erythema. After analysis of the video files by each reviewer, the number of mucosal breaks in each anonymous data file was determined by a consensual decision of the three reviewers.

\section{Detection of SNP with Smallest $p$ Value by GWAS}

The two groups of treated subjects were additionally divided into the following three groups based on increased numbers of small intestinal mucosal breaks after NSAIDs administration: (1) no injury group, subjects with no increase in small intestinal mucosal breaks after treatment; (2) mild injury group, subjects with an increase of one to four small intestinal mucosal breaks after treatment; (3) severe injury group, subjects with an increase of five or more small intestinal mucosal breaks after treatment. The three groups were compared in each treated group (loxoprofen group or celecoxib group) and in combined groups (loxoprofen group combined with celecoxib group) in the following combinations: (1) severe injury group compared with the no injury group; (2) severe injury group combined with the mild injury group compared with the no injury group; (3) severe injury group compared with the no injury group combined with the mild injury group.

The following subjects were excluded from analysis: (1) incomplete subjects with inadequate drug administration in the RCT; (2) subjects without evaluation of the entire small intestine by baseline capsule endoscopy; (3) subjects in which no increased mucosal breaks were detected because of failed evaluation of the entire small intestine by post-treatment capsule endoscopy.

\section{SNP Genotyping and Quality Control}

Genomic DNA was extracted from the peripheral blood leukocytes of 143 subjects using the QuickGene DNA whole blood kit L (Wako Pure Chemical, Osaka, Japan). In GWAS, the authors genotyped all samples using the Infinium OmniExpressExome-8 BeadChip (Illumina, San Diego, CA, USA) that contained a total of 951,117 SNPs. Samples with a call rate of $<0.99$, SNPs with a minor allele frequency $<0.01$, and SNPs with $p$ values less than the cutoff value $\left(p<1.0 \times 10^{-6}\right)$ for the Hardy-Weinberg equilibrium test as quality control filters were excluded. Among all SNPs in the BeadChips, 580,461 SNPs in the autosomal chromosomes were used for further analysis. Population stratification for the GWAS data was examined by principal component analysis (PCA) [11]. The genotype data of the samples along with European (CEU), African (YRI), and East Asian (JPT and CHB) individuals were obtained from the Phase II HapMap database. A quantilequantile plot ( $Q-Q$ plot) was drawn using observed $p$ values against expected $p$ values. After performing PCA, 16 cases and 123 controls were selected within the major Japanese cluster. Five SNPs showing the lowest $p$ value were validated using multiplex the PCR-based Invader assay.

\section{Genotype Imputation}

The gene with the SNP that had the lowest $p$ value was further analyzed by genotype imputation. Genotype imputation within the GWAS data was performed using the SHAPEIT2 and IMPUTE2 software in 250-kb regions on chromosome 20q11.21 [12]. The 1000 Genomes Project database (phase 3, October 2014) was used as a reference panel. We excluded SNPs that met the following criteria: minor allele frequency $<0.01$, Hardy-Weinberg equilibrium $p$ value $<1 \times 10^{-6}$, a low quality of imputation $\left(R^{2}<0.9\right)$, and a large allele frequency difference between the GWAS and reference panel (>0.16). 


\section{Statistical Analysis}

Numerical variables related to subject characteristics were compared by Student's t-test. Gender was compared by Fisher's exact test. Data are expressed as the mean value \pm SD. Association of SNPs with NSAID-induced small intestinal injury was evaluated by Fisher's exact test under three genetic models (additive, recessive, and dominant) using PLINK 1.90 software (http://pngu.mgh.harvard.edu/ purce 11/plink/). To characterize the population structure in the GWAS, PCA was performed using PLINK and R statistical environment version 3.0.1. A genetic inflation factor $\lambda(\lambda \mathrm{GC})$ was derived from $p$ values under the additive model obtained by PLINK. A quantile-quantile $(Q-Q)$ plot was drawn using the $\mathrm{R}$ program. To obtain an overview of the association of SNPs, a Manhattan plot of the study was generated using Haploview 4.2. Regional association plots were generated using LocusZoom [13].

\section{Results}

Of 150 subjects, 1 withdrew from drug administration, 2 had inadequate drug administration, 2 did not undergo evaluation of the entire small intestine in the baseline capsule endoscopy, and 2 had no mucosal breaks detected because of failed evaluation of the entire small intestine after capsule endoscopy following treatment and were excluded from analysis. In the GWAS, when we performed PCA using CEU, YRI, JPT, and CHB HapMap samples as references, we excluded four outlier samples that were not similar to the major Japanese cluster (Supplementary Figure S1A and S1B). Ultimately, 70 subjects in the loxoprofen group and 69 in the celecoxib group were analyzed.

In GWAS, we calculated $p$ values under three genetic models (additive, recessive, and dominant) using the 139 subjects. When a $Q-Q$ plot was generated, the $\underline{\lambda} \mathrm{GC}$ was 1.048 (Supplementary Figure S1C), indicating a low possibility of population stratification. The Manhattan plot of the minimum $p$ value with the three genetic models showed that there were no genetic loci surpassing genome-wide significance with the threshold $p$ value of $<5 \times 10^{-8}$ in all combinations of divided subgroups. The lowest $p$ value $(p=2.69$ $\times 10^{-7}$ with an odds ratio of 40.9) was detected comparing 16 cases (severe injury group) and 123 controls (no injury group combined with mild injury group) (Fig. 1). The characteristics of the two groups is shown in Table 1. Analyzing only 70 subjects treated with loxoprofen plus lansoprazole, the same SNPs had the lowest $p$ values in the GWAS ( $p=8.3$ $\times 10^{-6}$ with an odds ratio of 34.1 ).

Five SNPs (rs2070325, rs2889732, rs1169009, rs11696307, and rs11696310) showing the lowest $p$ value were located within the bactericidal/permeability-increasing fold-containing family B member 4 (BPIFB4) gene (Table 2 and Fig. 2). Of the five SNPs showing a linkage disequilibrium (LD) coefficient $\left(r^{2}\right)$ of 1.0, four were nonsynonymous SNPs and the remaining one was an intronic SNP (Table 2). To identify additional susceptible loci associated with NSAID-induced small intestinal injury, we conducted imputation analysis around the genomic region of chromosome 20q11.21 based on GWAS data and found that 23 SNPs within a $250-\mathrm{kb}$ region, including the BPIFB4 gene,

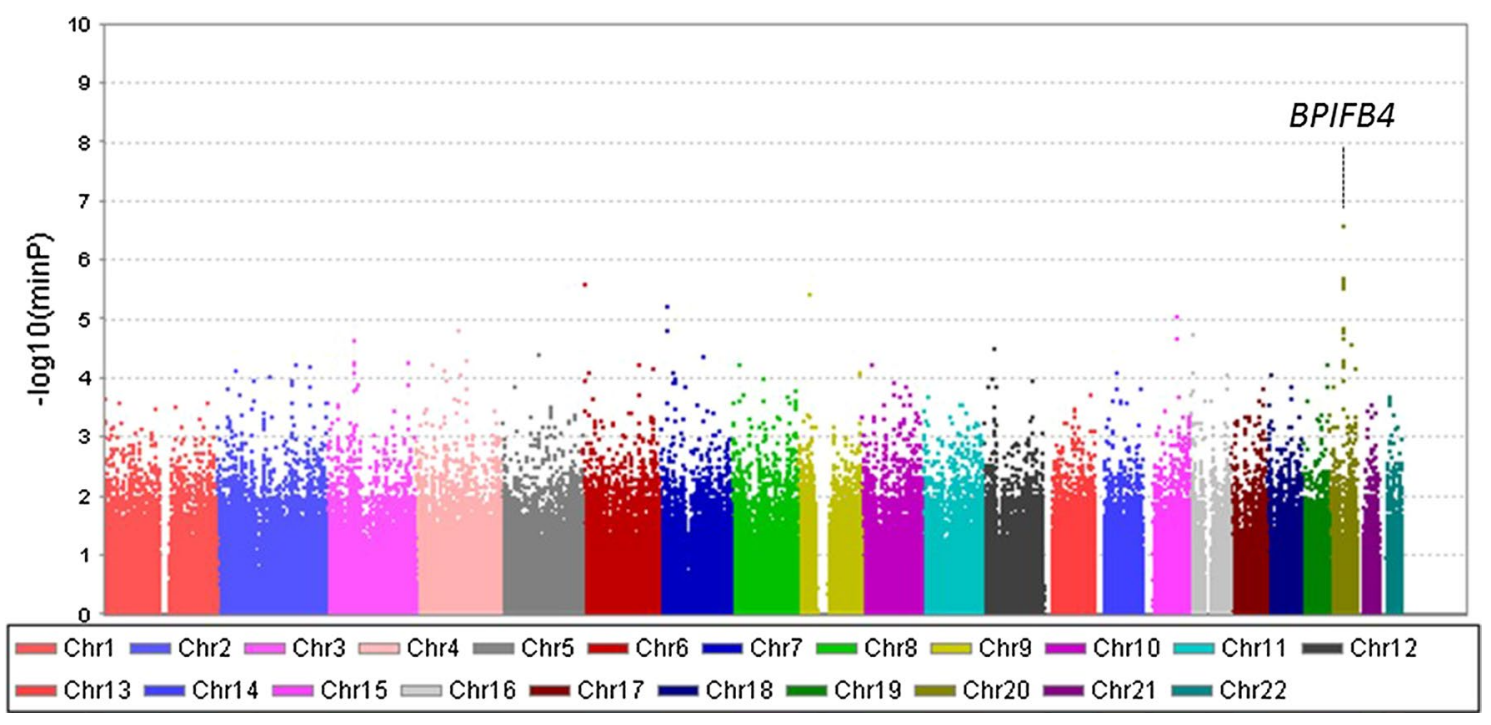

Fig. 1 Manhattan plot for GWAS of NSAID-induced small intestine mucosal breaks. The $y$-axis shows the $-\log 10$ of $p$ values of 580,461 SNPs. The minimum $p$ value under three genetic models (additive, recessive and dominant) was obtained by Fisher's exact test. The signal in the BPIFB4 region (20q11.21) is indicated 
Table 1 Characteristics of subjects compared between the severe injury group $(N=16)$ and no injury group combined with the mild injury group $(N=123)$

\begin{tabular}{llll}
\hline Small intestinal injury & $\begin{array}{l}\text { No and mild } \\
\text { injury group }\end{array}$ & Severe injury group & $p$ value \\
\hline Mucosal breaks & $0-4$ & 5 and more & \\
Number & 123 & 16 & \\
Age & $48.6 \pm 6.0$ & $48.9 \pm 7.6$ & 0.82 \\
Male/female & $58 / 65$ & $12 / 4$ & 0.060 \\
Weight $(\mathrm{kg})$ & $58.6 \pm 9.9$ & $64.2 \pm 14.0$ & 0.045 \\
BMI & $21.8 \pm 2.6$ & $22.6 \pm 3.3$ & 0.27 \\
Blood counts & & & \\
WBC ( $\mu \mathrm{l})$ & $6190 \pm 1630$ & $6680 \pm 1100$ & 0.25 \\
Hb (g/dl) & $13.9 \pm 1.3$ & $14.2 \pm 0.9$ & 0.29 \\
Plt (×10 $/ \mu l)$ & $22.7 \pm 4.6$ & $24.2 \pm 5.2$ & 0.25 \\
Blood chemistry & & & \\
TP (g/dl) & $7.0 \pm 0.4$ & $7.0 \pm 0.3$ & 0.83 \\
Alb (g/dl) & $4.5 \pm 0.2$ & $4.5 \pm 0.2$ & 0.60 \\
AST (IU/l) & $19.7 \pm 5.9$ & $29.3 \pm 5.5$ & 0.80 \\
ALT (IU/l) & $18.0 \pm 9.4$ & $17.2 \pm 7.9$ & 0.72 \\
$\gamma-G T P(I U / l)$ & $23.0 \pm 14.3$ & $28.6 \pm 21.7$ & 0.17 \\
BUN (mg/dl) & $11.1 \pm 2.3$ & $11.5 \pm 3.1$ & 0.25 \\
Cre (mg/dl) & $0.73 \pm 0.15$ & $0.81 \pm 0.13$ & 0.057 \\
GLU (mg/dl) & $93.9 \pm 5.9$ & $95.8 \pm 7.2$ & 0.24 \\
\hline
\end{tabular}

Numeral variables related to patient characteristics were compared between the celecoxib and loxoprofen groups by Student's $t$-test. Gender and the Helicobacter pylori infection rate were compared between the two groups by Fisher's exact test. Data are expressed as the mean value $\pm \mathrm{SD}$

showed almost complete $\mathrm{LD}\left(r^{2}>0.96\right)$ with the 5 SNPs and a suggestive association $\left(p<1 \times 10^{-6}\right.$, Supplementary Figure S2). Among these 23 SNPs, 4 (rs2424955, rs2424956, rs2424957, and rs2424958 within the intron of BPIFB4) showed the lowest $p$ value $\left(p=1.44 \times 10^{-7}\right.$ with an OR of 44.0, Supplementary Table S1).

\section{Discussion}

In the results of the GWAS, the SNP (rs2070325) with a minimum $p$ value $\left(p=2.69 \times 10^{-7}\right)$ was obtained by comparing 16 cases (severe injury group) and 123 controls (no injury group combined with mild injury group). The SNP was present in the gene of the BPIFB4 protein. We first identified the SNP, which was not detected in a previous candidate-gene approach for NSAID-induced small intestinal mucosal injury.

A total of five SNPs, including rs2070325, were in complete linkage disequilibrium $\left(r^{2}=1\right)$. Four of the five SNPs caused an amino acid substitution. In fact, three types of BPIFB4 may exist: the homozygous, heterozygous, and

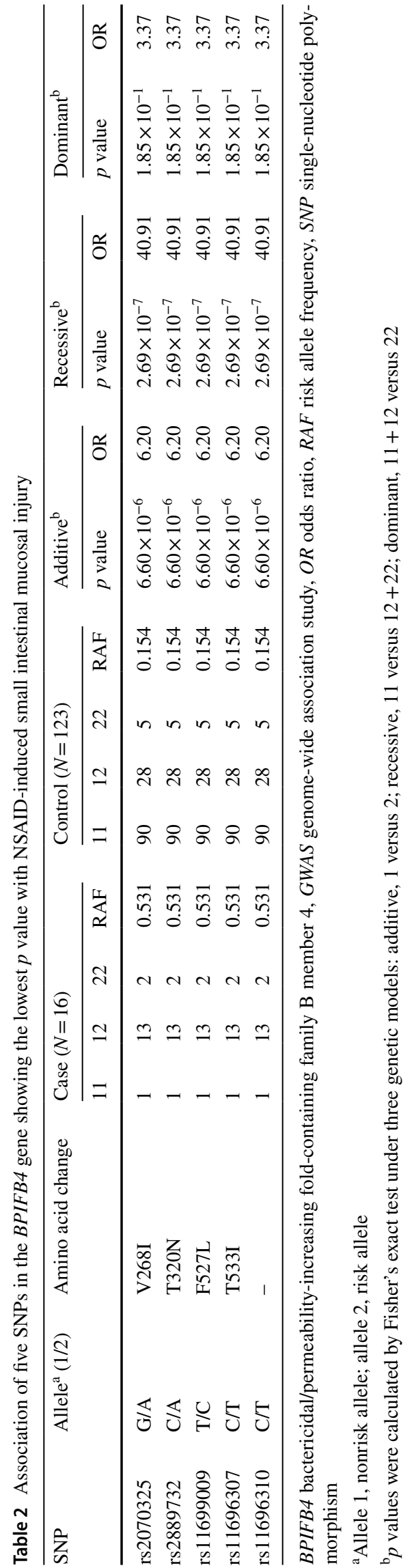


Fig. 2 Regional association plot around five SNPs showing the lowest $p$ value in the GWAS. The $-\log 10$ of the $p$ values (left $y$-axis) of the SNPs are shown according to their chromosomal position ( $x$-axis). The genomic recombination rates are represented by the blue line, and arrows indicate the locations of genes. The rs2070325 is represented as a purple circle, and the extent of linkage disequilibrium $\left(r^{2}\right)$ with the remaining SNPs is indicated by the color intensity. Five SNPs (rs2070325, rs2889732, rs11699009, rs11696307, and rs11696310) showing the lowest $p$ values are located within the BPIFB4 gene

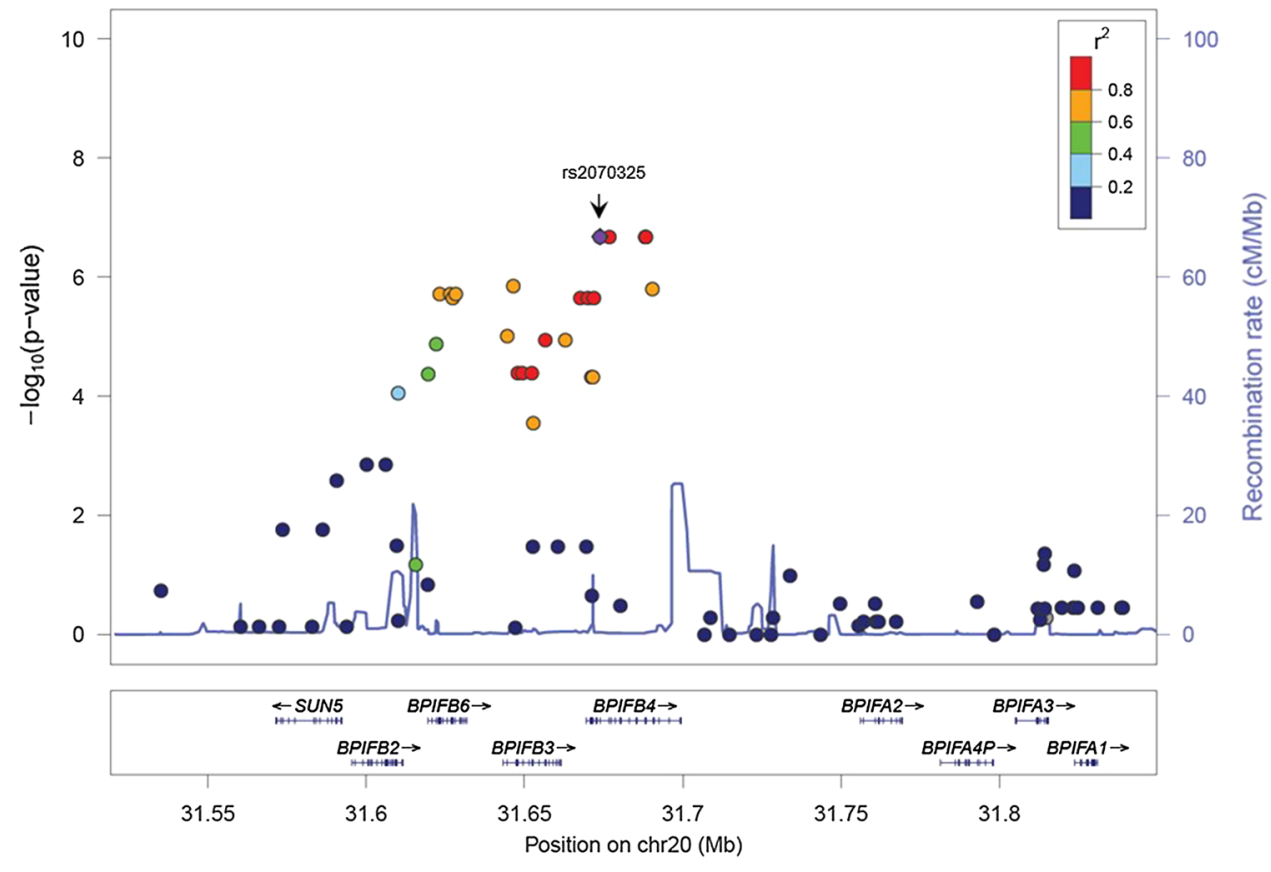

recessive homozygote of the NSAID risk type. Moreover, an additional 23 SNPs with $p<1 \times 10^{-6}$ were detected in BPIFB 4 by genotype imputation methods. Therefore, we hypothesized that SNPs in BPIFB4 are responsible for NSAID-induced small intestinal mucosal breaks.

The BPIFB4, which consists of 614 amino acids and has a molecular weight of $65,055 \mathrm{Da}$, is a bactericidal/permeability-increasing protein (BPI) [14-17]. BPI has antibiotic activity, endotoxin neutralization activity, and antiangiogenetic effects in its amino terminal and has opsonic activity and neutralizing lipopolysaccharide function in the carboxy terminal. BPI is produced and secreted by neutrophils in the mucosa and is distributed in the intramucosa, intraepithelial cells, and the surface of epithelial cells. The target of BPI is gram-negative bacteria. The cell wall of gram-negative bacteria is a double-membrane structure. The outer membrane is important to maintain the cell wall because gram-negative bacteria have a very thin peptidoglycan layer compared with gram-positive bacteria. The BPI invades the outer membrane of gram-negative bacteria to destroy it. In addition, gramnegative bacteria invading the BPI in the outer membrane have opsonic activity for ingestion by white blood cells. Furthermore, BPI neutralizes lipopolysaccharide function on the outer membrane of gram-negative bacteria [15, 16]. Briefly, BPI has strong antibiotic activity for gram-negative bacteria that invade and contact the mucosa, causing disinfection and detoxification.

The mechanism of NSAID-induced small intestinal mucosal injury is briefly considered as follows. First, permeability of the small intestinal mucosa increases. NSAIDs damage mitochondria in epithelial cells to weaken intercellular junctions between epithelial cells. Next, gut content, including bacteria and bile acids, invades the intramucosa and/or submucosa through the infinitesimal gap between epithelial cells [18, 19]. However, invasion of the gut content into mucosa is not sufficient to induce small intestinal mucosal breaks because strong intestinal mucosal immunity eliminates the invader. Prostaglandin (PG) is an important part of the mucosal defense to maintain mucosal blood flow and product mucus. PG is synthesized in response to cyclooxygenase (COX). NSAIDs inhibit COX to trigger a decrease in PG with the impairment of mucosal defense. In particular, inhibition of COX-1, which is constantly produced in diverse tissues for biologic defense, is most associated with small intestinal mucosal injury among the COXs $[3,19]$. Thus, the inhibition of COX-1 induces a decrease in PG in the mucosa to extend mucosal breaks with insufficient elimination of the invader [19]. Other than the above, there is a possibility that some components of bacteria including endotoxin are associated with NSAIDs-induced small intestinal mucosal injury. However, there are no reports to show a clear association between the two factors.

Bacteria are invaders because NSAID-induced small intestinal mucosal injury does not occur before anti-bacterial drug treatment [20]. SNPs in $B P I F B 4$, a member of the BPI family associated with the elimination of gram-negative bacteria, were associated with the risk of NSAID-induced small intestinal mucosal breaks in the study. Considering that gram-negative bacteria were intimately involved in NSAID-induced small intestinal mucosal injury, the results of the present study were rational from the aforementioned perspective. Recently, it was shown that PPIs influence small 
intestinal flora to exacerbate NSAID-induced enteropathy [21]. Our study may be influenced by PPIs. However, we propose that the significance of BPIFB4 for the small intestine is clear.

Recently, Villa et al. [22, 23] reported that BPIFB4 was detected by comparing long-living individuals with younger subjects in GWAS $\left(p<1 \times 10^{-4}\right)$. They also analyzed SNPs of BPIFP4 and reported that the four SNPs caused an amino acid substitution with complete linkage disequilibrium [24]. These four SNPs were the same as our SNPs. However, the allele frequency was inversed because of racial variation. Therefore, Ile229Val, Asn281Thr, Leu488Phe, and Ile494Thr in their study correspond to V268I, T320N, F527L, and T533I in our study, respectively. Because Japanese are known for the longevity of its people among others, inversed allele frequencies are consistent with their results. They used aging perspective in the analysis of BPIFB4 and reported that BPIFB4 was expressed in the CD34+ sub-fraction of circulating mononuclear cells, which is known to participate in vascular repair of ischemic tissues [22]. In their report, beneficial effects of BPIFB4 with the longevity-associated variant on endothelial function showed that the roles of $\mathrm{Ca}^{2+}$ mobilization and protein kinase $\mathrm{C}$ alpha in endothelial nitric oxide synthase activation and of endothelium-derived hyperpolarizing factor when endothelial nitric oxide synthase was inhibited [25]. We would like for these interesting results to be reevaluated in the view of antibacterial protein and repair of intestinal mucosa.

Although SNPs that surpassed the genome-wide significance level $\left(p<5 \times 10^{-8}\right)$ could not be identified through GWAS in the study, the results indicated that the SNPs of BPIFB4 were associated with NSAID-induced small intestinal mucosal injury because many SNPs on BPIFB4 had a low $p$ value. It was possible that the NSAID-induced small intestinal mucosal injury was complicated by a variety of factors, such as antibiotic proteins, drug metabolism, drug susceptibility, and intestinal bacterial flora [26]. The selection of the other SNPs may occur with future investigations.

In conclusion, the SNPs of BPIFB4 showed the lowest $p$ values in GWAS for NSAID-induced small intestinal mucosal injury. The effect of individual variability of the antibacterial protein on the expression enteropathy, whether involving the improvement of intestinal flora changed by drugs or drugs with an inhibitory effect on the antibacterial protein, was not specified. However, the results showed a possibility of the importance of antibacterial proteins for defending the intestinal mucosa. This study showed that SNPs of BPIFB4 may be useful for individual treatment for the defense of small intestinal mucosa from NSAIDs.

Acknowledgments The subjects of this study were based on our previous study financially supported by Pfizer Japan Inc. We thank Dr.
Takahisa Furuta, Hamamatsu University School of Medicine, for advising this study.

Author's contribution Study concept and design: SF, TM, and CS. Acquisition of data: SF, RH, MH, and TS. Analysis and interpretation of data: SF, KF, AT, TM, and MK. Drafting of the manuscript: SF, KF, AT, and TM. Critical revision of the manuscript for important intellectual content: AT, TM. KI, and CS. Statistical analysis: SF, KF, TM, and MK. Fund-raising: TM and CS. Study supervision: IK and CS.

Funding This study was financially supported by grants of the Project for Development of Innovative Research on Cancer Therapeutics and the Tailor-made Medical Treatment Program (BioBank Japan) funded by the Ministry of Education, Culture, Sports, Science, and Technology of Japan.

\section{Compliance with ethical standards}

Conflict of interest The authors declare no conflicts of interest.

Open Access This article is distributed under the terms of the Creative Commons Attribution-NonCommercial 4.0 International License (http://creativecommons.org/licenses/by-nc/4.0/), which permits any noncommercial use, distribution, and reproduction in any medium, provided you give appropriate credit to the original author(s) and the source, provide a link to the Creative Commons license, and indicate if changes were made.

\section{References}

1. Graham DY, Opekun AR, Willingham FF, et al. Visible smallintestinal mucosal injury in chronic NSAID users. Clin Gastroenterol Hepatol. 2005;3:55-59.

2. Maiden L, Thjodleifsson B, Theodors A, et al. A quantitative analysis of NSAID-induced small bowel pathology by capsule enteroscopy. Gastroenterology. 2005;128:1172-1178.

3. Goldstein JL, Eisen GM, Lewis B, et al. Video capsule endoscopy to prospectively assess small bowel injury with celecoxib, naproxen plus omeprazole, and placebo. Clin Gastroenterol Hepatol. 2005;3:133-141.

4. Goldstein JL, Eisen GM, Lewis B, et al. Small bowel mucosal injury is reduced in healthy subjects treated with celecoxib compared with ibuprofen plus omeprazole, as assessed by video capsule endoscopy. Aliment Pharmacol Ther. 2007;25:1211-1222.

5. Fujimori S, Takahashi Y, Seo T, et al. Prevention of traditional NSAID-induced small intestinal injury: recent preliminary studies using capsule endoscopy. Digestion. 2010;82:167-172.

6. Fujimori S, Seo T, Gudis K, et al. Prevention of nonsteroidal anti-inflammatory drug-induced small-intestinal injury by prostaglandin: a pilot randomized controlled trial evaluated by capsule endoscopy. Gastrointest Endosc. 2009;69:1339-1346.

7. Fujimori S, Takahashi Y, Gudis K, et al. Rebamipide has the potential to reduce the intensity of NSAID-induced small intestinal injury: a double-blind, randomized, controlled trial evaluated by capsule endoscopy. J Gastroenterol. 2011;46:57-64.

8. Furuta T, Sagehashi Y, Shirai N, et al. Influence of CYP2C19 polymorphism and Helicobacter pylori genotype determined from gastric tissue samples on response to triple therapy for $H$. pylori infection. Clin Gastroenterol Hepatol. 2005;3:564-573.

9. Pilotto A, Seripa D, Franceschi M, et al. Genetic susceptibility to nonsteroidal anti-inflammatory drug-related gastroduodenal 
bleeding: role of cytochrome $\mathrm{P} 4502 \mathrm{C} 9$ polymorphisms. Gastroenterology. 2007;133:465-471.

10. Fujimori S, Hanada R, Hayashida M, et al. Celecoxib monotherapy maintained small intestinal mucosa better compared with loxoprofen plus lansoprazole treatment: a double-blind, randomized, controlled trial. J Clin Gastroenterol. 2016;50:218-226.

11. Patterson N, Price AL, Reich D. Population structure and eigenanalysis. PLoS Genet. 2006;2:e190.

12. van Leeuwen EM, Kanterakis A, Deelen P, et al. Populationspecific genotype imputations using minimac or IMPUTE2. Nat Protoc. 2015;10:1285-1296.

13. Pruim RJ, Welch RP, Sanna S, et al. LocusZoom: regional visualization of genome-wide association scan results. Bioinformatics. 2010;26:2336-2337.

14. Levy O. A neutrophil-derived anti-infective molecule: bactericidal/permeability-increasing protein. Antimicrob Agents Chemother. 2000;44:2925-2931.

15. Schultz H, Weiss JP. The bactericidal/permeability-increasing protein (BPI) in infection and inflammatory disease. Clin Chim Acta. 2007;384:12-23.

16. Canny G, Levy O. Bactericidal/permeability-increasing protein (BPI) and BPI homologs at mucosal sites. Trends Immunol. 2008;29:541-547.

17. Bingle CD, Bingle L, Craven CJ. Distant cousins: genomic and sequence diversity within the BPI fold-containing (BPIF)/PLUNC protein family. Biochem Soc Trans. 2011;39:961-965.

18. Bjarnason I, Williams P, So A, Zanelli G, et al. Intestinal permeability and inflammation in rheumatoid arthritis: effects of non-steroidal anti-inflammatory drugs. Lancet. 1984;2:1171-1174.

19. Whittle BJ. Mechanisms underlying intestinal injury induced by anti-inflammatory COX inhibitors. Eur J Pharmacol. 2004;500:427-439.

20. Bjarnason I, Hayllar J, Smethurst P, et al. Metronidazole reduces intestinal inflammation and blood loss in non-steroidal antiinflammatory drug induced enteropathy. Gut. 1992;33:1204-1208.

21. Wallace JL, Syer S, Denou E, et al. Proton pump inhibitors exacerbate NSAID-induced small intestinal injury by inducing dysbiosis. Gastroenterology. 2011;141:1322.

22. Villa F, Carrizzo A, Spinelli CC, et al. Genetic analysis reveals a longevity-associated protein modulating endothelial function and angiogenesis. Circ Res. 2015;117:333-345.

23. Spinetti G, Sangalli E, Specchia C, et al. The expression of the BPIFB4 and CXCR4 associates with sustained health in longliving individuals from Cilento-Italy. Aging. 2017;9:370-380.

24. Vecchione $\mathrm{C}$, Villa F, Carrizzo A, et al. A rare genetic variant of BPIFB4 predisposes to high blood pressure via impairment of nitric oxide signaling. Sci Rep. 2017;7:9706.

25. Spinelli CC, Carrizzo A, Ferrario A, Villa F, et al. LAV-BPIFB4 isoform modulates eNOS signalling through $\mathrm{Ca}^{2+} / \mathrm{PKC}$-alphadependent mechanism. Cardiovasc Res. 2017;113:795-804.

26. Bjarnason I, Scarpignato C, Holmgren E, et al. Mechanisms of damage to the gastrointestinal tract from non-steroidal anti-inflammatory drugs. Gastroenterology. 2018;154:500-514. 